\title{
Storm watchers
}

\section{The turbulent history of weather prediction from Franklin's kite to El Niño}

\author{
John D. Cox
}

$\mathrm{L}$ 'auteur est un journaliste scientifique renommé qui a reçu une formation en météorologie et en océanographie au MIT. Son livre est, en quelque sorte, une histoire de la prévision du temps, de la fin du XVIII ${ }^{\mathrm{e}}$ siècle à la fin du $\mathrm{XX}^{\mathrm{e}}$. Cette histoire est complètement biaisée puisque l'auteur se focalise essentiellement sur les États-Unis, mais son parti pris est souvent riche d'informations. Il raconte cette histoire en 28 chapitres courts (le livre a 252 pages) qui sont chacun le roman d'une tranche de vie d'un des pionniers de notre science, avec une description toujours intéressante du milieu dans lequel elle se déroule. On aurait pu s'attendre à une suite d'hagiographies, mais ce n'est vraiment pas le cas. L'auteur tord le cou à un certain nombre de héros d'une façon des plus réjouissantes, même s'il perpétue un certain nombre de poncifs.

La première partie du livre est consacrée aux précurseurs, avec Benjamin Franklin, Luke Howard et James Glaisher. On y apprend en particulier que Franklin était passionné par le climat et que Howard regrettait d'avoir fait trop de latin au cours de ses études.

La deuxième partie porte sur les tempêtes américaines et notamment sur celle qui a opposé William Redfield et James Espy ; elle présente aussi Elias Loomis, Joseph Henry, Matthew Maury et William Ferrel. C'est la biographie de Maury qui est la plus originale. Celui qui est généralement présenté comme un grand homme y est traité de charlatan (le mot est d'Alexander Bache) à l'aide de citations extraites du livre de Marcel Brillouin, Mémoires originaux sur la circulation générale de l'atmosphère (Carré, 1900), qui n'est pas cité dans la bibliographie.

Viennent ensuite les premières tentatives de prévision du temps avec Robert FitzRoy, Urbain Le Verrier,
Cleveland Abbe, John Finley, Mark Harrington, Isaac Cline, Gilbert Walker et LeRoy Mesinger. C'est l'occasion pour l'auteur de montrer le conflit incessant qui oppose les tenants d'une météorologie pratique à ceux pour qui la recherche doit précéder les applications ; il a conduit à la suppression des prévisions après le suicide de FitzRoy, mais aussi à l'interruption des recherches sur les tor-
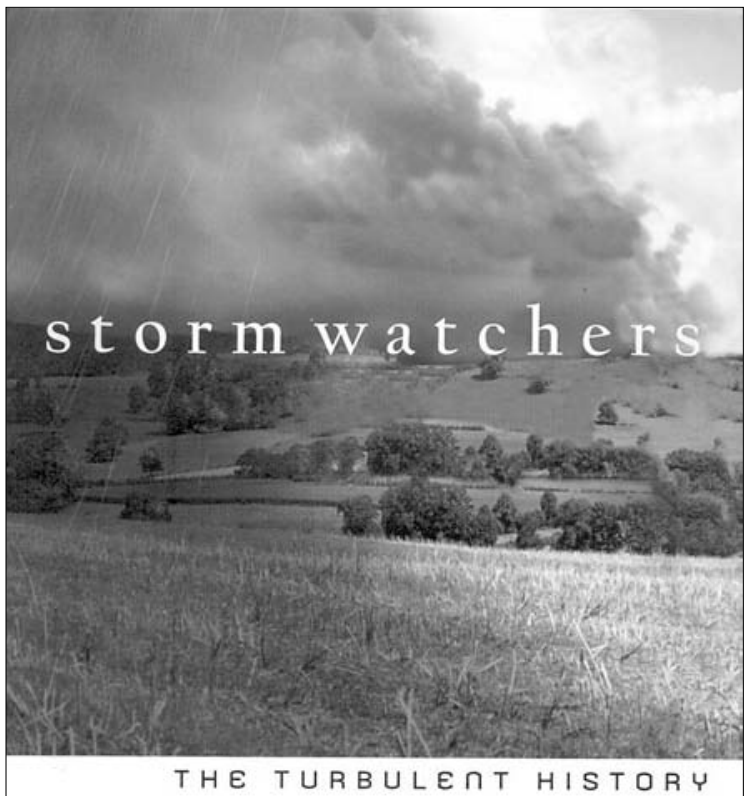

OF UEATHER PREDICTION FROM

FRAOKLIN'S KITE TO EL TIÑO

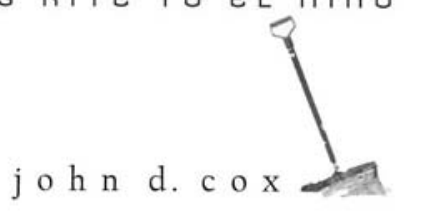

La quatrième partie est celle du début de la réconciliation entre la théorie et la pratique avec Vilhelm Bjerknes, Lewis Richardson, Jacob Bjerknes, Tor Bergeron, Carl-Gustav Rossby et Sverre Petterssen. On lira avec grand intérêt les errements de certains météorologistes américains au cours de la préparation du débarquement en Normandie et le chapitre sur Rossby où sont contées son opposition avec le Weather Bureau et la fondation de la première entreprise privée en météorologie.

La cinquième partie nous introduit dans l'ère actuelle de la prévision avec Jule Charney, Jerome Namias, Edward Lorenz, Theodore Fujita et Ants Leetmaa. Cox raconte avec verve la journée du 17 juin 1997 où Leetmaa, directeur du Climate Prediction Center des États-Unis, a tenu une conférence de presse pour annoncer l'El Niño du siècle pour l'hiver suivant et les remous que cette annonce a provoqués. Je regrette qu'il n'ait pas raconté le coup de poker de Charney qui a fait accepter la prévision numérique au Weather Bureau sur la base d'une prévision réussie, celle de la tempête du President's Day. Et pourtant, une fois en service opérationnel, le modèle s'est révélé très décevant et a dû rapidement être remplacé par un bon « vieux » modèle barotrope. nades aux États-Unis à la fin des années 1880 , le mot ayant même été banni du vocabulaire officiel jusqu'en 1952. Le cyclone qui a ravagé Galveston en 1900 est le sujet principal du chapitre sur Cline, qui réussit le tour de force de faire croire qu'il avait prévu ce cyclone, ce qui était faux, alors qu'il avait même affirmé qu'aucun cyclone ne pourrait jamais toucher cette ville. Une médaille Isaac Cline récompense encore les employés du National Weather Service ayant rendu des services exceptionnels...
C'est un livre passionnant, on y apprend une grande quantité de choses sur l'histoire de la prévision du temps.

Michel Rochas

Storm watchers.

The turbulent history of weather prediction from Franklin's kite to El Niño.

Par John D. Cox.

John Wiley.

Hoboken, 2002, 252 p. 